This material is published in the open archive of Mid Sweden University

DIVA http://miun.diva-portal.org to ensure timely dissemination of scholarly and technical work. Copyright and all rights therein are retained by authors or by other copyright holders. All persons copying this information are expected to adhere to the terms and constraints invoked by each author's copyright. In most cases, these works may not be reposted without the explicit permission of the copyright holder.

Damghanian, M.; Olsson, R.; Sjöström, M., "Performance analysis in Lytro camera: Empirical and model based approaches to assess refocusing quality," in 2014 IEEE International Conference on Acoustics, Speech and Signal Processing (ICASSP), pp.559-563, 4-9 May 2014 http://dx.doi.org/10.1109/ICASSP.2014.6853658

(C) 2015 IEEE. Personal use of this material is permitted. Permission from IEEE must be obtained for all other uses, in any current or future media, including reprinting/republishing this material for advertising or promotional purposes, creating new collective works, for resale or redistribution to servers or lists, or reuse of any copyrighted component of this work in other works." 


\title{
PERFORMANCE ANALYSIS IN LYTRO CAMERA: EMPIRICAL AND MODEL BASED APPROACHES TO ASSESS REFOCUSING QUALITY
}

\author{
Mitra Damghanian, Roger Olsson, and Mårten Sjöström \\ Department of Information and Communication Systems, Faculty of Science, Technology and Media \\ Mid Sweden University, Sundsvall, Sweden
}

\begin{abstract}
In this paper we investigate the performance of Lytro camera in terms of its refocusing quality. The refocusing quality of the camera is related to the spatial resolution and the depth of field as the contributing parameters. We quantify the spatial resolution profile as a function of depth using empirical and model based approaches. The depth of field is then determined by thresholding the spatial resolution profile. In the model based approach, the previously proposed sampling pattern cube (SPC) model for representation and evaluation of the plenoptic capturing systems is utilized. For the experimental resolution measurements, camera evaluation results are extracted from images rendered by the Lytro full reconstruction rendering method. Results from both the empirical and model based approaches assess the refocusing quality of the Lytro camera consistently, highlighting the usability of the model based approaches for performance analysis of complex capturing systems.
\end{abstract}

Index Terms- Plenoptic capture, camera evaluation, resolution analysis

\section{INTRODUCTION}

Cameras have a wide range of properties to suit diverse applications. Proper evaluation of the camera performance is then necessary to illustrate the capabilities and limitations of the unconventional cameras. Plenoptic cameras, with the aid of computational photography techniques and the increase in computational power, bring new possibilities to the area of image capturing and hence providing new applications. To make a plenoptic camera, a lenslet array structure is introduced to a conventional camera system to empower capturing the directional information $[1,2]$.

In this paper we investigate the performance of Lytro camera, which is the first plenoptic camera introduced to the consumer market, in terms of its refocusing quality. Since introduction of the Lytro camera, it has dragged attentions as a

This work has been supported by grant 2012/0328 of the KK Foundation, Sweden, grant 00156702 of the EU European Regional Development Fund, Mellersta Norrland, Sweden, and by grant 00155148 of Länsstyrelsen i Västernorrland, Sweden. smart device $[3,4]$ and has even become the subject for performance analysis and reverse engineering attempts [5]. A detailed performance analysis of Lytro camera as a pioneer plenoptic camera will help to put this device in perspective with other plenoptic products as well as the user expectations.

Refocusing quality of the plenoptic camera is a direct function of the camera's spatial resolution and depth of field. A larger depth of field will provide a wider depth range in which the refocusing can be applied, and a better spatial resolution in the depth plane of interest will result in a higher quality reconstructed image.

Empirical measurements can provide the ground truth for desired camera performance parameters such as spatial resolution. However the rendering approaches also influence the results. Model based approaches remove the hassle of the practical measurements and they skip the rendering step leading to a direct method to extract camera performance parameters such as spatial resolution, though the challenge would be accuracy and the level of details the models can provide. In this context, models with low complexity but capable of extracting high level of details are appreciated.

The sampling pattern cube (SPC) model has been proven useful in representation and evaluation of plenoptic cameras $[6,7]$. Spatial resolution in a plenoptic camera is influenced by both the captured ray information and the focal properties of the capturing system. The SPC model carries both the ray sampling as well as the focus properties of the capturing systems and hence is suitable to be utilized for extracting high level camera properties such as resolution terms.

In this paper, the utilized method for camera performance analysis will be discussed in Section 2. Section 3 will give the details about the SPC model based approach to extract camera performance parameters. The experimental setup for the empirical measurements will be explained in Section 4. Results will be provided and briefly discussed in Section 5 and finally Section 6 concludes the work.

\section{METHOD}

The lateral resolution profile throughout various depth planes is a valuable indicator of the performance of the plenoptic 


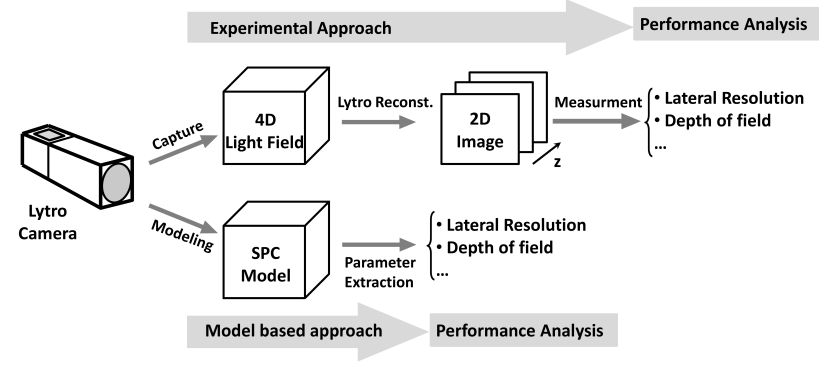

Fig. 1. Illustration of the experimental and the model based methods for camera evaluation

camera, which empowers us to make a reasonable comparison between various camera structures with different capture parameters. The measured lateral resolution of a plenoptic camera depends both on the capture parameters and the utilized rendering method.

Here we investigate the spatial resolution profile of the camera as a function of the object distance to the cameras aperture. Figure 1 illustrates the method for evaluation of the Lytro camera using the empirical and the model based approaches.

For the scope of this work, the lateral resolution $R$ for a complex plenoptic capturing system is defined as the inverse of the minimum distance between two resolvable points $x_{1 z}$ and $x_{2 z}$ located at a specific depth plane $z$ perpendicular to the optical axis, which after normalization to the sensor pixel pitch $p$ gives:

$$
R_{n}=\frac{p}{\min \left[x_{1 z}-x_{2 z}\right]} .
$$

The depth plane of interest can be located in the system's common field of view $(\mathrm{CFoV})$ or be visible for only a number of lens elements in the system.

The depth of field $D o F$ is defined as the depth range in which the rendered image is sufficiently sharp with respect to a resolution threshold Rth:

$$
D o F=D_{f a r}-D_{\text {near }} .
$$

Thus the Dof is closely related to the resolution threshold that separates the depth ranges which give sharp (in focus) and blur (out of focus) images. It is important to have the same resolution threshold when comparing the DoF between different capturing systems.

\section{MODEL BASED APPROACH}

Table 3 summarize the geometrical camera parameters to generate the SPC model (as described in $[6,7]$ ) of the Lytro camera. Figure 2 describes the Lytro camera system. The SPC model of a capturing system contains the object side tip position and the span of the cone shaped light samples recorded on
Table 1. Utilized geometrical parameters for modeling the Lytro camera

\begin{tabular}{lc}
\hline Lenslet focal length, $f$ & $25 \mu \mathrm{m}$ \\
Lenslet pitch, $D$ & $13.9 \mu \mathrm{m}$ \\
Lenslet working f-number, $N$ & 1.8 \\
Pixel pitch, $p$ & $1.39 \mu \mathrm{m}$ \\
Number of lenslets horizontally & 328 \\
The gap size, $g$ & $25.1 \mu \mathrm{m}$ \\
Main lens focal length, $F$ & $6.4 \mathrm{~mm}$ \\
Main lens working f-number & 1.92 \\
\hline
\end{tabular}

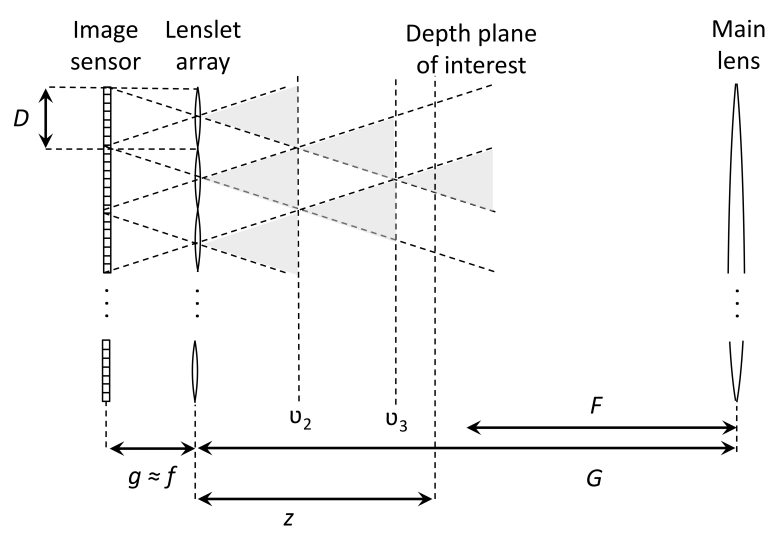

Fig. 2. Lytro camera geometry

the image sensor pixels. The SPC model then uses this information to extract camera parameters such as lateral resolution throughout depth [7]. In the current SPC model implementation of the Lytro camera, it is enough to look into the model of the system for a single line of pixels behind a single line of lenslets to extract the lateral resolution profile, since the geometry of the lytro camera is shift invariant considering the periodicity of the camera structure.

The SPC model based lateral resolution extractor defined in [7] is valid for the area within the CFoV of the capturing system. To use the same extractor for the depth ranges outside the $\mathrm{CFoV}$, visible only by a limited number of the lenslets (which is an interesting depth range in conventional plenoptic cameras such as Lytro configuration), we use an incremental methodology. In this method we start from the lenslet plane and use the SPC based lateral resolution extractor applied to a single lenslet structure for the depth ranges between the lenslet plane (see Figure 2) and the depth plane where the $\mathrm{CFoV}$ of a two lenslet system starts (shown by $\nu_{2}$ in Figure 2). We continue the same approach progressively for the whole number of lenslets in a row and so we will cover the depth range of interest starting from the plane of the lenslets.

For comparison purposes, an analytical approach [8] is also employed to calculate the lateral resolution values in the 
Lytro camera system. This analytical approach is based on the resolution in a single lens system and is then extended to plenoptic cameras by dividing the single lens resolution by the number of lenslets that image a single point in the depth plane of interest. For a conventional plenoptic camera system (the case of the Lytro camera), this analytical approach defines the effective resolution ratio $\epsilon_{P}$ (the ratio between the effective resolution $R_{e}$ and the sensors resolution $R_{t}$ ) as:

$$
\epsilon_{P}=\frac{R_{e}}{R_{t}}=\frac{g}{z}\left(\frac{p}{\max \left(|D \cdot g / z|, p, s_{\lambda}\right)}\right),\left|\frac{z}{g}\right| \geq 1
$$

where the effective resolution $R_{e}$ is defined as the inverse of the blur size $s$ for the depth plane of interest and the diffraction limit $s_{\lambda}$ is defined as:

$$
s_{\lambda}=1.22 \lambda N,
$$

$\lambda$ is the wavelength and $N$ is the f-number of the lens. Obtained $\epsilon_{P}$ values are then comparable with the normalized resolution $R_{n}$ from the SPC model based approach.

\section{EXPERIMENTAL SETUP}

The experimental setup incorporates a Lytro camera capturing so called light field photos, a scene with an ISO 12233 test chart [9], and a motorized Cinevate Atlas 30 camera slider. The distance between the camera and the test chart is varied from $17 \mathrm{~mm}$ to $838 \mathrm{~mm}$, the far end restricted by the camera slider length. A set of 30 so called light field photos (LRF) is acquired using the Lytro camera at uniformly spaced depths. The camera is operated with fixed zoom and focus in so called Everyday Mode. Refocused image stacks are produced from each LRF using the Lytro Desktop Software. The image in each stack exhibiting the highest sharpness is selected as having a focus plane coinciding with the test chart and used in subsequent resolution analysis by measuring the systems modulation transfer function (MTF) as set out in [9]. Both MTF30 and MTF50 is used, where MTFN correspond to the spatial frequency of the MTF equal to a contrast of $\mathrm{N} \%$ relative to that of the MFT at spatial frequency 0 . The MTF50 closely resembles the perceived sharpness of an image whereas the MTF30 renders the analysis less influenced by the sharpening operation that Lytro Desktop performs after refocusing each image, which would be the case for the more commonly used MTF50. Compared to MTF50, the MTF30 also provides a closer match with the vanishing resolution definition used in the model based and the analytical approach. A closer correspondence would be provided by MTF10 at the expense of a more noise sensitive resolution measurement, which is undesirable given the lossy compression artifacts arising in the refocused image stack provided by Lytro Desktop.

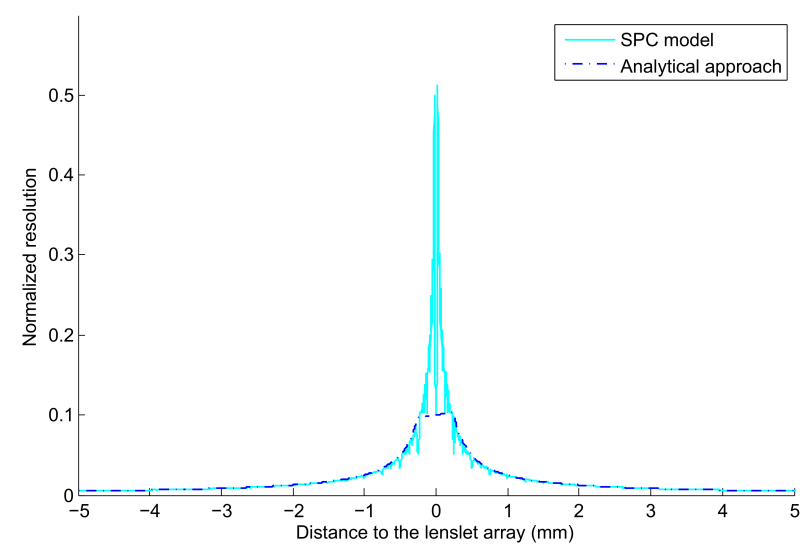

Fig. 3. Spatial resolution of the lenslet array system through depth obtained from analytical and SPC model based methods

\section{RESULTS AND DISCUSSION}

Figure 3 illustrates the spatial resolution of the lenslet array system for Lytro camera obtained from the SPC model based approach. Results are normalized according to the image sensor pixel size, so a resolution equal to 1 means the full sensor resolution. The SPC model based results predict the spatial resolution values equal to the number of lenslets at the plane of the lenslets since two points are defined resolvable if they contribute to two different light cones in the SPC model of the camera and the base area of light cones is covering the lenslet pitch at this depth plane. Then the SPC model predicts resolution peaks at planes with the depth equal to the focal length of the lenslets followed by a dominating resolution drop behavior. Predicted peaks in the resolution graph reflect the evenly distribution of the light cone base areas in that depth plane.

Figure 3 also illustrates the results for the spatial resolution values obtained from the analytical approach (see [8] for the details). This approach calculates the resolution in the plenoptic camera by approximating the lateral resolution of the plenoptic system as the lateral resolution of a single lenslet divided by the number of lenslets imaging a specific 3D point. This simplifying assumption causes a lower level of details in the results such as not predicting planes where the resolution drops. As a result this method smoothes out the details in, and provides an estimate of, the effective lateral resolution ratio. In comparison, the SPC model gives lateral resolution values with a higher level of details. Spatial resolution values from both the SPC model and the analytical model agree in their general trend after the depth plane $\nu_{10}$.

Figure 4 presents the MTF30 and MTF50 of the Lytro camera when the distance between the test chart and the camera is varied. The MTF graphs follow the trend predicted by the SPC model and the analytical method. Note that the main lens shifts and stretches the resolution graphs towards optical infinity such that the decaying effect of the right side of the 


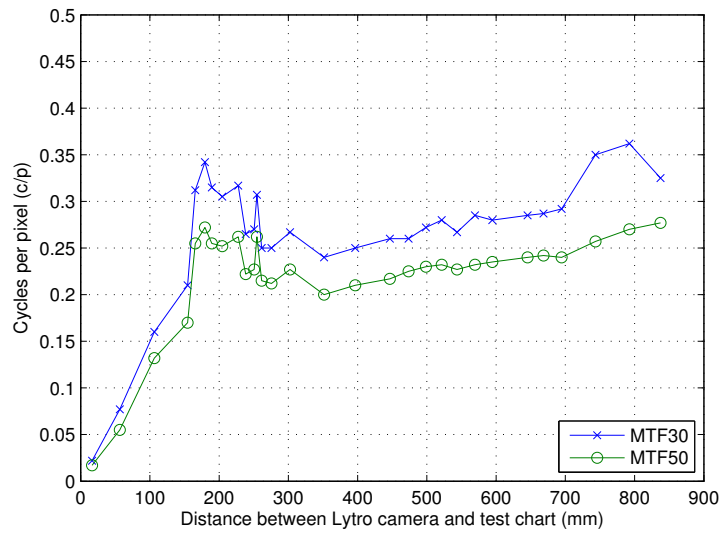

Fig. 4. Measured MTF30 and MTF50 as a function of distance between the test chart and the camera

graph in Figure 3 falls outside the evaluated distance range. MTF30 more closely approaches the vanishing resolution definition used in the other analysis methods, but at the expense of larger variations in the measurement results. Comparing different approaches for extracting the lateral resolution and depth of field of the Lytro camera, the model based approach has the advantage of predicting parameters of interest quicker and with less effort than empirical approaches.

The SPC model based results predict existence of depth planes with better resolution than the number of lenslets (values bigger than 0.1 in Figure 3). A similar effect is also present in the empirical results with values exceeding $328 / 1080 \approx 0.304 \mathrm{~mm}$ in Figure 4 . A contributing factor to the exceeding values from resolution measurements is also the image sharpening applied by the Lytro Desktop software to the refocusing images. A sharpening that can be seen as an overshoot in individual MTF graphs, i.e. with contrast values within the MTF exceeding the contrast at spatial frequency 0 . To what degree these exceeding values stem from postprocessing sharpness relative to confirmation of SPC model's prediction requires more detailed investigations.

Setting an explicit detailed correspondence between the image side and object side resolution values requires the knowledge about the relative distance between the main lens system and the lenslet array. Lytro camera is using a $6.4 \mathrm{~mm}$ lens. The knowledge about where the image plane of the main lens system is located will determine the object side DoF of the camera. Figure 5 shows the model based results for $D o F$ (corresponding to $\epsilon_{P}=0.1$ ) in the Lytro camera as a function of distance between lenslets and the main lens. The best $D o F$ is obtained when the relative distance between the main lens and the lenslet plane is set to the focal length of the main lens plus $\nu_{10}$. At this relative distance $D_{\text {near }}$, which is the depth of the nearest in-focus plane relative to the camera objective, is calculated to be $8.8 \mathrm{~cm}$ which corresponds to the point $G=6.65 \mathrm{~mm}$ in Figure 5 .

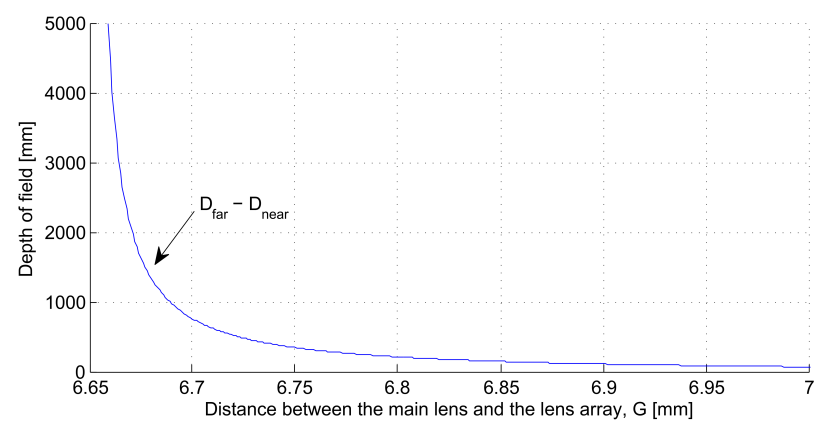

Fig. 5. DoF for $\epsilon_{P}=0.1$ as a function of the distance between the main lens and the lenslet array

To get a wider depth of field from the Lytro camera, the distance between the lenslet array and the main lens system should be set very close, ideally equal, to the focal length of the main lens system. This setting corresponds to the Everyday capturing mode, which will map the high resolution depth range around the lenslet array to a wider depth range in the object space in front of the camera. In contrast, for a shallower depth of field, it is desired to make the distance between the lenslet array and the main lens system bigger so that the high resolution depth range around the lenslet array plane is translated to a narrow depth range in the object space centered on the objects of interest. This setting is provided in the Lytro cameras Creative Mode.

A benefit of using model based approaches over the empirical resolution analysis of plenoptic cameras is the lack of effects from the mandatory rendering algorithms used when producing e.g. refocused images. Basically, the SPC modeling entirely skips this rendering step when extracting lateral resolution and so the camera system performance will be directly evaluated rather than a combination of capture and rendering steps. Such reliable camera performance analysis results will then be useful in comparing different camera systems regardless of their utilized image rendering methods, as well as forming the reference resolution relative to which different rendering methods may be evaluated.

\section{CONCLUSION}

Performance of the Lytro camera was investigated using empirical, analytical and model based approaches. To assess the refocusing quality of the camera, the lateral resolution profile and the depth of field from both empirical and model based approaches were analyzed. Results from both approaches indicate the wide depth of field that is expected from the Lytro camera, being a conventional plenoptic camera when operating in Everyday Mode. The SPC approach generated lateral resolution and depth of field results comparable to empirical analysis, confirming the usability of model based approaches for performance analysis of complex capturing systems. 


\section{REFERENCES}

[1] Gabriel Lippmann, "Epreuves reversibles. photographies integrals," Comptes-Rendus Academie des Sciences, vol. 146, pp. 446-451, 1908.

[2] Ren Ng, Marc Levoy, Mathieu Brédif, Gene Duval, Mark Horowitz, and Pat Hanrahan, "Light field photography with a hand-held plenoptic camera," Computer Science Technical Report CSTR, vol. 2, 2005.

[3] Gary Anthes, "Smarter photography," Commun. ACM, vol. 55, no. 6, pp. 16-18, June 2012.

[4] Rachel Ehrenberg, "The digital camera revolution: Instead of imitating film counterparts, new technologies work with light in creative ways," Science News, vol. 181, no. 2, pp. 22-25, 2012.

[5] Todor Georgiev, Zhan Yu, Andrew Lumsdaine, and Sergio Goma, "Lytro camera technology: theory, algorithms, performance analysis," in IS\&T/SPIE Electronic Imaging. International Society for Optics and Photonics, 2013, pp. 86671J-86671J.

[6] Mitra Damghanian, The Sampling Pattern Cube: A Framework for Representation and Evaluation of Plenoptic Capturing Systems, Ph.D. thesis, Mid Sweden University, 2013.

[7] Mitra Damghanian, Roger Olsson, and Mårten Sjöström, "Extraction of the lateral resolution in a plenoptic camera using the SPC model," in 3D Imaging (IC3D), 2012 International Conference on, 2012, pp. 1-5.

[8] Christian Perwass and Lennart Wietzke, "Single lens 3D-camera with extended depth-of-field," in IS\&T/SPIE Electronic Imaging. International Society for Optics and Photonics, 2012, pp. 829108-829108.

[9] "Photography - Electronic still-picture cameras - Resolution measurements," 2000. 